Zusammenfassung der Dissertation mit dem Titel

\title{
„Die Stellungnahme der Zielgesellschaft und die Gegenleistung in der Unternehmensübernahme ${ }^{6}$
}

Dissertation vorgelegt von Philip Schmoll

Erstgutachter: Prof. Dr. Dres. h.c. Werner F. Ebke

Zweitgutachter: Priv.-Doz. Dr. Sebastian Mock

Institut für deutsches und europäisches Gesellschafts- und Wirtschaftsrecht 
Gegenstand der Arbeit war die Stellungnahme zu Art und Höhe der Gegenleistung nach $\S 27$ Abs. 1 Satz 2 Nr. 1 Wertpapiererwerbs- und Übernahmegesetz (,WpÜG“) samt der hiermit zusammenhängenden Vor-, Rand- und Folgefragen. Die zentralen Kernthemen der Arbeit waren dabei die Stellungnahme der Zielgesellschaft als Anlass einer Unternehmensbewertung sowie die Sanktionierung fehlerhafter Stellungnahmen aus europarechtlicher Sicht.

\section{Problemaufriss und Anlass der Arbeit}

Ein öffentliches Angebotsverfahren unter dem WpÜG stellt für die Zielgesellschaft ein einschneidendes Ereignis dar. Die Aktionäre der Zielgesellschaft entscheiden als Angebotsadressaten kollektiv über den Erfolg des öffentlichen Angebots und damit über eine Veränderung der Aktionärs-/Kontrollstruktur in der Zielgesellschaft. Vorstand und Aufsichtsrat als Leitungsorgane der Zielgesellschaft kommt dabei die wohl bedeutendste Pflicht zur Unterstützung dieser Entscheidung zu. Im Rahmen der Stellungnahme nach $§ 27$ WpÜG sind sie berechtigt und verpflichtet, die Angebotsadressaten über Art und Höhe der angebotenen Gegenleistung zu informieren ( $\$ 27$ Abs. 1 Satz 2 Nr. 1 WpÜG). Dieser Teil der Stellungnahme (die „Finanzielle Stellungnahme“) ist für die Angebotsadressaten von besonderer Bedeutung und birgt für die Leitungsorgane besondere Schwierigkeiten und Probleme.

Art und Höhe der Gegenleistung sind für die Entscheidung der Angebotsadressaten über die Annahme des Angebots häufig ausschlaggebend. Wirtschaftlich denkende Angebotsadressaten haben beim Verkauf ihrer Aktien regelmäßig ausschließlich ein Interesse an der Realisierung eines höchstmöglichen Profits (return on investment). Offeriert der Bieter folglich eine angemessene Gegenleistung, wird das Angebot aller Voraus-sicht nach von einer Vielzahl der Angebotsadressaten angenommen werden. Ist die Gegenleistung zu niedrig, werden die Angebotsadressaten hingegen wohl von der Annahme absehen und ihre Aktien behalten. Um sich über die Gegenleistung zu informieren, werden viele Aktionäre unter anderem die Stellungnahme der Zielgesellschaft nach § 27 WpÜG zu Rate ziehen. Die Leitungsorgane der Zielgesellschaft vermögen durch ihre Sachnähe zur Zielgesellschaft die Gegenleistung am besten einzuschätzen, zumal sie über intime Kenntnisse der gegenwärtigen Finanz-, Vermögens- und Ertragslage der Zielgesellschaft und deren zukünftigen Entwicklungsmöglichkeiten verfügen. Dies befähigt sie dazu, eine fundierte Stellungnahme zu Art und Höhe der gebotenen Gegenleistung abzugeben. Im Hinblick auf den Ausgang des Angebotsverfahrens sind die Informationen zur Gegenleistung somit die wichtigste Komponente der Stellungnahme gemäß § 27 WpÜG. Nicht ohne Grund wird dieser Teil daher als „Kern“ der Stellungnahme der Zielgesellschaft bezeichnet.

Den Anlass zur Arbeit gaben die Verwaltungspraxis der Bundesanstalt für Finanzdienstleistungsaufsicht („BaFin“) sowie die „Richtlinie 2004/25/EG des Europäischen Parlaments und des Rates betreffend Übernahmeangebote“ (,EU-Übernahmerichtlinie“):

Die BaFin sah Anlass, ihre langjährige Verwaltungspraxis im Zusammenhang mit Stellungnahmen von Zielgesellschaften zu veröffentlichen. Ziel sei es, Klarheit und Rechtssicherheit für die Leitungsorgane der Zielgesellschaft zu schaffen. Hierzu stellte die BaFin allgemeine 
Maßstäbe zur Erstellung von Stellungnahmen nach $\S 27$ WpÜG auf. Danach besteht ein zunehmender Bedarf an der Erläuterung und Konkretisierung der Pflichten, die den Leitungsorganen aus $\S 27 \mathrm{WpÜG}$ erwachsen. Die veröffentlichten Vorgaben der BaFin zur Stellungnahme der Zielgesellschaft vermitteln zwar, wie die BaFin die Anforderungen des $\S 27$ WpÜG im Sinne ihrer Verwaltungspraxis beurteilt, die Aussagen der BaFin zur Stellungnahme zu Art und Höhe der Gegenleistung sind aber sehr knapp und allgemein gehalten. Die Gegenleistung dürfe danach nicht ohne Begründung als ,angemessen“ bezeichnet werden. Zwar sei nicht anzugeben, wie die Höhe die Gegenleistung aus Sicht der Leitungsorgane ausgestaltet sein müsste. Die Leitungsorgane haben aber Gründe zu nennen, warum sie die gebotene Gegenleistung nicht für angemessen halten. Die BaFin gibt damit lediglich zu verstehen, dass die Stellungnahme zur Gegenleistung zu begründen ist, wobei die Wiedergabe einer hypothetischen Gegenleistung (sog. Wertindikation) nicht erforderlich sei. Zu den Bewertungspflichten und der Stellungnahme zur Art der Gegenleistung schweigt die BaFin hingegen gänzlich. Die Frage, ob ein konkreter Wert der Zielgesellschaft oder nur eine Wertspanne zu bestimmen ist, klingt zwar an (,weder feste Gegenleistung noch Spanne“ ), wird im Ergebnis aber offen gelassen.

Auf Rechtsfolgenseite gab die EU-Übernahmerichtlinie Anlass zur Untersuchung. Im Kapitalmarktrecht hat der Gesetzgeber grundsätzlich die Möglichkeit, Rechtsverstöße mit Mitteln des Strafrechts, des Öffentlichen Rechts und des Privatrechts zu sanktionieren. In der Praxis veranlasst die BaFin bei sog. fehlerhaften Stellungnahmen häufig, die bereits veröffentlichte Stellungnahme der Zielgesellschaft zu ergänzen bzw. zu korrigieren. Zusätzlich zu dieser Pflicht können die Leitungsorgane für fehlerhafte Stellungnahmen durch die BaFin mit einer Geldbuße belegt werden. Im Gegensatz zur Angebotsunterlage muss die Veröffentlichung der Stellungnahme der Zielgesellschaft nicht von der BaFin gestattet werden (vgl. § 14 Abs. 2 WpÜG). Das Fehlen der Gestattungspflicht birgt ein hohes Risiko für die Leitungsorgane der Zielgesellschaft, die erforderliche Form nicht einzuhalten. Zudem besteht - anders als für fehlerhafte Angebotsunterlagen nach $\S 12 \mathrm{WpÜG} \mathrm{-} \mathrm{keine} \mathrm{ausdrückliche,} \mathrm{privatrechtliche}$ Haftungsnorm für fehlerhafte Stellungnahmen. Privatrechtliche Haftungsfälle sind nicht bekannt. Das Fehlen einer privatrechtlichen Haftungsnorm lässt dagegen im Hinblick auf eine effektive Normdurchsetzung des § 27 WpÜG aufhorchen. Vor diesem Hintergrund wird die derzeitige Sanktionierung fehlerhafter Stellungnahmen insbesondere mit Blick auf das Unionsrecht kritisiert, das gewisse Anforderungen an die vom Mitgliedsstaat gewählten Sanktionen stellt.

\section{Überblick über den Aufbau der Arbeit}

Der erste Teil der Arbeit widmet sich den Vorfragen zur finanziellen Stellungnahmen. Zunächst werden hier die nationale und europäische Entwicklung der (finanziellen) Stellungnahme behandelt (Erster Teil, §§ 1, 2). Da das Unionsrecht ebenfalls eine Stellungnahme der Zielgesellschaft vorsieht, stellen sich zudem europarechtliche Vorfragen, die in $\S 3$ des ersten Teils untersucht werden. Im Mittelpunkt des ersten Teils steht indes der allgemeine Pflichtenmaßstab von $\S 27$ WpÜG (Erster Teil, § 4), der die Grundlage für den Hauptteil der 
Arbeit bildet. Schließlich wurde in $\S 5$ des Ersten Teils der Zeitrahmen der Stellungnahme nach $\S 27$ Abs. 3 WpÜG genauer analysiert.

Der zweite Teil der Arbeit stellt den Hauptteil der Arbeit dar. In diesem Teil werden die konkreten Vorstands- und Aufsichtsratspflichten im Rahmen der finanziellen Stellungnahme behandelt (Zweiter Teil [Vorstand]; Dritter Teil [Aufsichtsrat]). Zunächst werden die Vorstandspflichten in Bezug auf die Stellungnahme zur Art der Gegenleistung behandelt (Zweiter Teil, § 1). Im zweiten Teil des Hauptteils werden dann die Vorstandspflichten hinsichtlich der Stellungnahme zur Höhe der Gegenleistung untersucht (Zweiter Teil, § 2). Wegen der anfangs erwähnten Bedeutung steht dieser Teil der Stellungnahme im Mittelpunkt der Arbeit. Der Hauptteil schließt mit einer Einordnung der finanziellen Stellungnahme in die sog. Handlungsempfehlung der Leitungsorgane (Zweiter Teil, § 3).

Im Nachgang des Hauptteils werden schließlich Folge- und Randfragen zur finanziellen Stellungnahme abgehandelt. Problematisch ist etwa eine etwaige Verschwiegenheitspflicht der Leitungsorgane (Vierter Teil, § 1). Weiterhin war auf die Rolle externer Berater und deren sog. Fairness Opinions einzugehen (Vierter Teil, §§ 2, 3). Ein weiterer Schwerpunkt der Arbeit wird in $\S 4$ des vierten Teils erörtert: die bereits angerissene Problematik der Sanktionierung fehlerhafter Stellungnahmen. Die Arbeit schließt mit einer Zusammenfassung der wesentlichen Ergebnisse, einer abschließenden Würdigung sowie einer Praxisempfehlung (Fünfter Teil).

\section{Thesen der Arbeit}

Die Stellungnahme der Leitungsorgane zu Art und Höhe der angebotenen Gegenleistung ist das Kernelement der Stellungnahme nach $\S 27$ WpÜG. Für die Angebotsadressaten ist dieser Teil der Stellungnahme von herausragender Bedeutung. Nicht selten wird ihre Entscheidung über das Angebot auf diesen Informationen gestützt werden. Aussagen zur Gegenleistung waren daher schon vor Einführung des WpÜG ein wesentlicher Bestandteil öffentlicher Angebote. Erfreulich ist, dass die Stellungnahme zu Art und Höhe der Gegenleistung ebenso im Zuge des WpÜG Eingang in die obligatorischen Teile des $§ 27$ Abs. 1 Satz 2 WpÜG gefunden hat. Europarechtlich stellt diese Regelung zwar eine inhaltliche Übererfüllung der EU-Übernahmerechtlinie dar, ist die Übererfüllung aber wegen des mindestharmonisierenden Charakters der Richtlinie unbedenklich. Gleichwohl ergeben sich hieraus Besonderheiten für die Auslegung (Stichwort: richtlinienorientierte Auslegung) und die Vorlagefähigkeit an den EuGH.

Für die Leitungsorgane der Zielgesellschaft birgt die Stellungnahme zu Art und Höhe der Gegenleistung besondere Anforderungen und Schwierigkeiten. Bei ihrer Pflichterfüllung haben Vorstand und Aufsichtsrat neben den kapitalmarktrechtlichen Grundsätzen ebenso die allgemeinen gesellschaftsrechtlichen Maßstäbe zu beachten. Dies ist der rechtlichen Doppelnatur der Stellungnahme gemäß § 27 WpÜG geschuldet. Die Besonderheit des Bewertungsanlasses liegt zudem im engen Zeitrahmen, in dem die Leitungsorgane der Zielgesellschaft gegenleistungsbezogene Aussagen in der Stellungnahme zu treffen haben. Vorstand 
und Aufsichtsrat haben die Stellungnahme nach § 27 Abs. 3 WpÜG gleichermaßen unverzüglich zu veröffentlichen, d.h. innerhalb von zwei Wochen nach Übermittlung der Angebotsunterlage.

Die Stellungnahme zur Art der angebotenen Gegenleistung unterscheidet sich in ihrer Komplexität je nach der Angebotsart. Offeriert der Bieter im Rahmen eines Übernahme-/Pflichtangebots eine Geldleistung in Euro je Aktie der Zielgesellschaft, ergeben sich in der Regel keine besonderen Pflichten der Leitungsorgane. Werden hingegen Tauschaktien angeboten, ist jedenfalls auf die Zulässigkeit des Tauschangebots sowie auf die gesetzlichen Vorgaben an die Tauschaktie (Liquidität, Marktzulassung und Stimmrecht) einzugehen. Besondere Bedeutung kommt dabei die zukunftsgerichtete Aussage über die Liquidität der Tauschaktie zu. Die Leitungsorgane der Zielgesellschaft haben hierbei auf die erwartete Annahmequote des Tauschangebots, das erwartete Abgabeverhalten der (ins-besondere institutionellen) Anleger, die historischen durchschnittlichen Handelsvolumina, die Existenz größerer Aktienpakete, die Streuung der Aktien im Publikum (vgl. etwa $\S 9$ BörsZulV) sowie auf die Aufnahmebereitschaft der Aktionäre einzugehen. Bei einfachen Erwerbsangeboten hängt die Informationsintensität/-komplexität von der Art der angebotenen Gegenleistung ab (höher bei Geldleistung in Fremdwährung oder Tauschaktien; niedriger bei Geldleistung in Euro).

Die Stellungnahme der Zielgesellschaft zur Höhe der Gegenleistung ist ein Anlass für eine Unternehmensbewertung. Im AktG und im WpÜG steht bei Bewertungsanlässen der Terminus der „Angemessenheit“ im Mittelpunkt. So verlangt § 31 Abs. 1 WpÜG eine angemessene Gegenleistung, § 305 Abs. 1 AktG eine angemessene Abfindung, und § 327a Abs. 1 AktG eine angemessene Barabfindung. Lediglich im Wortlaut des $§ 27$ WpÜG findet sich dies nicht wieder. Vorstand und Aufsichtsrat haben gleichwohl auch in der Stellungnahme zur Höhe der Gegenleistung deren Angemessenheit zu prüfen. Zur Höhe der Gegenleistung kann nämlich nur dann wertend Stellung bezogen werden, wenn man die vom Bieter angebotene Gegenleistung mit dem Wert der Aktie ins Verhältnis setzt - im Ergebnis also die Angemessenheit der Gegenleistung bewertet. Die Angemessenheit im Sinne des § $27 \mathrm{WpÜG}$ war dabei durch Auslegung zu ermitteln. Auf den ersten Blick mag es naheliegen, hierfür den Angemessenheitsbegriff aus $\S 31$ WpÜG heranzuziehen. Dann wären Vorstand und Aufsichtsrat der Zielgesellschaft allerdings lediglich verpflichtet, den sich daraus ergebenden gesetzlichen Mindestpreis zu prüfen. Für diese Prüfung kann je-doch gerade nicht die der Stellungnahme immanente Sachnähe der Organe zur Zielgesellschaft fruchtbar gemacht werden. Denn der gesetzliche Mindestpreis basiert ausschließlich auf Marktpreisen wie dem Drei-Monats-Durchschnittskurs der Aktie sowie Vorerwerben von Aktien durch den Bieter. Gleichwohl erfordert die Stellungnahme zur Höhe der Gegenleistung bei Übernahme/Pflichtangeboten zunächst eine Prüfung der gesetzlichen Mindestpreisvorschriften.

Zur Beurteilung der Angemessenheit ist jedoch vielmehr der sogenannte Normwert des $\S 27$ WpÜG zu ermitteln. Dieser Normwert gibt dabei rechtlich vor, mit welchen Bewertungsmethoden die Organe der Zielgesellschaft den Wert der Aktie ökonomisch zu bestimmen haben. Die erforderlichen Bewertungsmethoden ergeben sich dabei aus einer Analyse der Stellung- 
nahme als Bewertungsanlass. Strukturell ist hier zwischen dominierten und nicht dominierten Bewertungsanlässen zu differenzieren. In Abgrenzung zu den Bewertungsanlässen des Unternehmensvertrags und des Squeeze-Outs ist die Stellungnahme zur Höhe der Gegenleistung als nicht-dominierter Bewertungsanlass zu qualifizieren. Ein nicht-dominierter Bewertungsanlass zeichnet sich dadurch aus, dass (1) die Deinvestitionsentscheidung des Aktionärs von Freiwilligkeit geprägt ist, (2) kein zwangsweiser Eingriff durch den Bieter bzw. den Mehrheits- oder Hauptaktionär erfolgt, sowie (3) kein zwangsweiser Verlust von Aktieneigentum oder Mitgliedschaftsrechten mit dem Übernahmeangebot einhergeht. Der Bieter kann eine Veränderung der Eigentumsverhältnisse bzw. der Rechtsverhältnisse an den Aktien der Zielgesellschaft nicht alleine und ein-seitig durchsetzen. Schließlich nehmen Vorstand und Aufsichtsrat nicht zu einer Abfindung, sondern zu einer Gegenleistung Stellung. Im Ergebnis ist die Stellungnahme der Zielgesellschaft daher auch kein Instrument zur Bestimmung einer Gegenleistung bzw. Abfindung, sondern die Würdigung und Meinung zu einer Gegenleistung, die von einer anderen Partei angeboten wird. Der Normwert des $§ 27$ WpÜG ist daher nicht analog zu den Bewertungs-grundsätzen bei aktienrechtlichen Strukturmaßnahmen zu bestimmen. Das heißt, dass - unabhängig von dem ohnehin engen Zeitrahmen - bereits strukturell kein Ertragswertverfahren nach IDW 1 erforderlich ist.

Vielmehr haben Vorstand und Aufsichtsrat der Zielgesellschaft eine Unternehmensbewertung im Schnelldurchgang durchzuführen. Hierbei haben sie nach ihrem Ermessen geeignete und übliche Bewertungsmethoden auszuwählen. Statt der exklusiven Durchführung eines Ertragswertverfahrens nach IDW S 1 sind mehrere kapitalmarkt- und kapitalwertorientierte Bewertungsmethoden nebeneinander und gleichrangig anzuwenden. Durch diese Methodenpluralität ergibt sich als Normwert des § 27 WpÜG kein konkreter Wert, sondern eine Wertbandbreite, in welche dann die angebotene Gegenleistung einzustellen ist.

Die angebotene Gegenleistung ist ,,angemessen“ im Sinne des $§ 27$ WpÜG, wenn sie innerhalb oder oberhalb einer Wertbandbreite liegt, die auf Grundlage mehrerer anerkannter Bewertungsmethoden (unter anderem DCF-Verfahren, Börsenkursanalyse, Vorerwerbspreise, Analystenmeinungen) zu ermitteln ist. Der Börsenkurs gilt als erster Anhaltspunkt, ist jedoch vor dem Risiko ineffizienter Aktienmärkte nicht überzubewerten. Im Rahmen der Begründung der Stellungnahme gilt grundsätzlich $\S 2$ Nr. 3 WpÜGAngebV analog, wobei eine sog. Wertindikation nicht verpflichtend ist. Offeriert der Bieter Tauschaktien als Gegenleistung, haben die Leitungsorgane die Aktie der Zielgesellschaft und die Tauschaktie möglichst nach dem Grundsatz der Methodengleichheit zu bewerten. Die angebotene Gegenleistung ist bei einem Tauschangebot, ,angemessen“ im Sinne des $\S 27$ WpÜG, wenn das angebotene Umtauschverhältnis innerhalb oder oberhalb einer Wertbandbreite von ermittelten Umtauschverhältnissen liegt. Bei einfachen Erwerbsangeboten sind bei der Pflichtenquantität und qualität keine Abstriche zu machen. Die Angemessenheit der Gegenleistung kann unter anderem mit der (theoretischen) Einhaltung der gesetzlichen Mindestpreisvorschriften begründet werden. 
Die ermittelten Ergebnisse aus der finanziellen Stellungnahme müssen zwar in der grundsätzlich erforderlichen Handlungsempfehlung berücksichtigt werden, darf sich die Empfehlung aber nicht ausschließlich an den Interessen der Angebotsadressaten ausrichten. Bei der Berücksichtigung der Angemessenheit der Gegenleistung in der Handlungsempfehlung ist nicht, wie in der Praxis üblich, die Angemessenheit im Sinne des § 31 Abs. 1 WpÜG maßgeblich, sondern die Angemessenheit im Sinne des § 27 WpÜG. Ebenfalls ist der Praxis neutraler Stellungnahmen Einhalt zu gebieten, die ihre Enthaltung zum Angebot zunehmend und rechtswidrig mit gegenleistungsbezogenen Argumenten begründen.

Die Pflichten des Aufsichtsrats sind mit den Vorstandspflichten weder identisch noch besteht eine bloße Kontrollpflicht. Der Aufsichtsrat hat unter Zugriff auf die vom Vorstand ermittelten Informationen eine eigene Beurteilung der Art und Höhe der Gegenleistung vorzunehmen. Stimmen Aufsichtsrat und Vorstand in der Beurteilung überein, können sie eine gemeinsame Stellungnahme veröffentlichen. Gehen die Beurteilungen auseinander, können den Aufsichtsrat zusätzliche (Informationsermittlungs-)Pflichten treffen. Vorstand und Aufsichtsrat unterliegen schließlich gleichermaßen der allgemeinen gesellschaftsrechtlichen Geheimhaltungspflicht nach $\S \S 93$ Abs. 1 Satz 3, 116 Satz 1 Aktiengesetz (,AktG“).

Zunehmende Bedeutung für die Finanzielle Stellungnahme erlangen externe Berater, zu deren Einbeziehung die Leitungsorgane lediglich verpflichtet sind, wenn die genannten Pflichten nicht eigenständig erfüllt werden können. Regelmäßig werden im Zuge einer finanziellen Stellungnahme sog. Fairness Opinions von externen Beratern eingeholt, die eine Vielzahl von Funktionen erfüllen können. Eine Fairness Opinion unterscheidet sich insbesondere von einem Bewertungsgutachten nach IDW S 1. Das sog. Transaktionsinstrument bezweckt gerade nicht die Preisfindung, sondern ist lediglich eine Beurteilung der Angemessenheit eines bereits festgelegten Angebotspreises anhand mehrerer Bewertungs-methoden (Grundsatz der Methodenvielfalt/-gleichheit). Hierin liegt die wesentliche Gemeinsamkeit mit der finanziellen Stellungnahme, weshalb die Stellungnahme der Leitungsorgane zur Angemessenheit der Gegenleistung als „Fairness Opinion der Zielgesellschaft“ bezeichnet werden kann. Inzwischen bestehen zwei Berufsgrundsätze (DVFA und IDW S 8), die Empfehlungen zur Erstellung von Fairness Opinions aufstellen und zu deren Standardisierung beitragen. Im Umgang mit Fairness Opinions treffen die Leitungsorgane zudem zusätzliche Pflichten (unter anderem Auswahl des externen Beraters, Überprüfung der Fairness Opinion).

Hinsichtlich der Sanktionierung fehlerhafter Stellungnahmen ist Ausganspunkt der Untersuchung, dass die Organe der Zielgesellschaft nach herrschender Meinung für die Veröffentlichung einer fehlerhaften Stellungnahme gegenüber den Aktionären haften nur aus $\S 826$ Bürgerliches Gesetzbuch und $\S 117$ Abs. 1 Satz 1 AktG. In der Praxis werden diese Ansprüche allerdings in aller Regel an Beweisproblemen auf der Vorsatzseite dieser Normen scheitern. Eine eigene Haftungsnorm mit Beweiserleichterungen wie für fehlerhafte Angebotsunterlagen sieht das WpÜG für fehlerhafte Stellungnahmen nicht vor. Damit sind fehlerhafte Stellungnahmen privatrechtlich praktisch sanktionslos. Der deutsche Gesetzgeber vertraut bis dato auf eine exklusiv öffentliche-rechtliche Sanktionierung des $\S 27$ WpÜG durch die Bußgeldvorschriften in $\S 60 \mathrm{WpÜG}$. Diese Sanktionierung hat dem in Art. 17 der EU- 
Übernahmerichtlinie kodifizierten Grundsatz der Effektivität zu entsprechen. Danach haben die Mitgliedsstaaten alle geeigneten Maßnahmen zu treffen, um die Durchsetzung der einzelstaatlichen Vorschriften zu gewährleisten. Die Sanktionen müssen hier-zu wirksam, verhältnismäßig und abschreckend sein.

Den Mangel an einer abschreckenden Wirkung hat der deutsche Gesetzgeber durch das Zweite Finanzmarktnovellierungsgesetz behoben. Durch die zum 1. Januar 2018 in Kraft getretene Änderung der Bußgeldvorschrift nach § 60 WpÜG wurde neben der Erhöhung des Höchstbetrags der Geldbuße für eine fehlerhafte Stellungnahme zudem ein neuer Abs. 5 eingefügt. Danach kann die Ordnungswidrigkeit mit einer Geldbuße bis zum Zweifachen des aus dem Verstoß gezogenen wirtschaftlichen Vorteils geahndet werden. Diese neue Regelung vermag jedenfalls das Merkmal der „Abschreckung“ zu erfüllen. In Verbindung mit der erheblichen Erhöhung der Geldbußen ist diese Regelung geeignet, Vorstand und Aufsichtsrat der Zielgesellschaft vor Rechtsverstößen gegen § 27 WpÜG abzuhalten. Denn durch die Regelung kann der Vorteil aus einer fehlerhaften Stellungnahmen nicht mehr gegenüber einer berechenbaren und gedeckelten Geldbuße überwiegen. Den Organmitgliedern wird dadurch der Anreiz genommen, Sonderzahlungen aus einer sogenannten golden parachuteKlausel bzw. durch den Bieter gewährte Vorteile für die Förderung der Übernahme mit einer potentiellen Geldbuße abzuwägen.

Zwar würde eine privatrechtliche Haftungsnorm für fehlerhafte Stellungnahmen eine weitaus höhere Abschreckungswirkung haben, würden die Schäden aus Haftungsansprüchen von Aktionären wohl deutlich über dem in Abs. 5 angedrohten Bußgeld liegen. Bei der Sanktionsauswahl steht den Mitgliedsstaaten europarechtlich aber ein weites Ermessen zu. Die Entscheidung des deutschen Gesetzgebers für eine ausschließlich öffentlich-rechtliche Durchsetzung dürfte dahingehend jedenfalls innerhalb seines Ermessens bewegen. Außerhalb seines Ermessens bewegt sich der deutsche Gesetzgeber jedoch bei der wirksamen Sanktionierung fehlerhafter Stellungnahmen. Wirksam ist die Sanktionierung nach Rechtsprechung des EuGH nur, wenn die Durchsetzung der Regelung bzw. die Verwirklichung des Regelungsziels gewährleistet ist.

Durch die exklusive öffentlich-rechtliche Durchsetzung des $§ 27$ WpÜG wird der informatorische Schutz durch die Stellungnahme indes gerade nicht erfüllt. Vor ihrer Veröffentlichung wird die Stellungnahme, anders als die Angebotsunterlage, durch die BaFin oder einen Dritten weder gebilligt noch geprüft. Damit besteht ein hohes Risiko, dass das Übernahmeangebot auf Grundlage fehlerhafter Informationen in der Stellungnahme angenommen bzw. nicht angenommen wird. Angebotsadressaten, die das Angebot vor etwaigen Ergänzungen oder Ausbesserungen der Stellungnahme angenommen haben, können von der Annahme nicht mehr zurücktreten. Nach ihrer Veröffentlichung besteht zudem eine begrenzte Entdeckungswahrscheinlichkeit von Fehlern in der Stellungnahme. Zum einen folgt dies aus dem begrenzten Zeitfenster der BaFin-Prüfung. In der Regel beträgt die Annahmefrist in der Praxis vier oder fünf Wochen ab Veröffentlichung der Angebotsunterlage. Da die Stellungnahme erst nach 14 Tagen ab Beginn der Annahmefrist veröffentlicht wird, hat die BaFin bis zum Ende der Annahme-frist folglich nur zwei oder drei Wochen Zeit, etwaige Fehler 
aufzudecken. Zum anderen bestehen regelmäßig personelle und finanzielle Engpässe bei der BaFin.

Die Stellungnahme der Zielgesellschaft steht zudem keiner nachträglichen gerichtlichen oder sachverständigen Überprüfung offen. Das Prüfungsdefizit während des Übernahmeangebots wird also auch nicht durch eine materielle Inhaltskontrolle der Stellungnahme ex post kompensiert. Ferner besteht ein Bedürfnis zum Ausgleich von Schäden durch den mangelnden Informationsschutz einer fehlerhaften Stellungnahmen. Zwar gewährt der Mindestpreis nach § 31 WpÜG einen gewissen Vermögensschutz. Der Mindestpreis basiert aber gerade nur auf Marktpreisen, weshalb dieser Preis vom angemessenen Wert der Aktien abweichen kann. Die Stellungnahme zur Höhe der Gegenleistung soll eine solche Abweichung offenlegen. Ist die Stellungnahme allerdings fehlerhaft, können Schäden entstehen, die nicht durch eine privatrechtliche Haftung aufgefangen werden.

Schließlich ist der primärrechtliche Grundsatz der Äquivalenz verletzt. Eine fehlerhafte Angebotsunterlage wird im Vergleich zu einer fehlerhaften Stellungnahme mit mehr als doppelt so hohen Geldbußen sowie einer privatrechtlichen Haftung sanktioniert. Als intendiertes Gegenstück und Spiegelbild zur Angebotsunterlage wird eine fehlerhafte Stellungnahme aber nicht annährend spiegelbildlich und somit auch nicht annährend äquivalent sanktioniert. Das Unionsrecht gebietet folglich ein „Private Enforcement“ des $\S 27 \mathrm{WpÜG.} \mathrm{Die} \mathrm{de} \mathrm{lege} \mathrm{lata}$ europarechtswidrige Sanktionierung ist durch eine europarechtskonforme Sanktionierung de lege ferenda zu korrigieren, indem eine $\S 12 \mathrm{WpÜG}$ entsprechende Haftungsnorm für fehlerhafte Stellungnahmen in das WpÜG eingeführt wird. 